\title{
Velocidad de operación de vehículos ligeros en curvas de carreteras convencionales
}

\author{
Elena García-Jiménez \\ Grupo de Investigación en Ingeniería de Carreteras (GIIC), Universtitat Politècnica de \\ València
Ana María Pérez-Zuriaga, Francisco Javier Camacho-Torregrosa, David Llopis- Castelló, Alfredo García \\ Cargo, Empresa o institución, País
}

\section{RESUMEN}

La velocidad de operación es uno de los factores más estudiados en el análisis de la seguridad vial por su relación con la siniestralidad. En la fase de explotación es relativamente sencillo calcular la velocidad de operación a partir de datos empíricos, pero, en las fases de planeamiento y de proyecto, sólo será posible estimarla.

Numerosos autores han modelizado la velocidad de operación de vehículos ligeros en curvas de carreteras convencionales considerando su velocidad mínima. Algunos a partir de observaciones en aquellos puntos de la carretera más significativos como el punto medio de la curva (velocidad mínima), otros a partir de datos continuos utilizando, por ejemplo, dispositivos GPS. La mayoría de los modelos que estiman esta velocidad se basan únicamente en el percentil 85 de la distribución o en su velocidad media. Además, la mayoría de los estudios asumen que sus datos se distribuyen según una normal sin, en muchos casos, comprobar dicha hipótesis.

El objetivo de este estudio es analizar la distribución de la velocidad desarrollada por vehículos ligeros circulando en condiciones de flujo libre en curvas de carreteras convencionales. Para ello, la investigación se basa en determinar si los datos de velocidad de 63 curvas disponibles de una base de más de $16.000 \mathrm{vh} \cdot \mathrm{km}$ se distribuyen según una normal, y en el análisis del comportamiento de la media y la desviación típica de la distribución de velocidades en curvas.

Se ha concluido que la distribución normal no es la que mejor se ajusta al comportamiento de la velocidad en la mayoría de los casos.

\section{INTRODUCCIÓN}

La seguridad vial es uno de los principales aspectos a considerar en la ingeniería de carreteras por el elevado número de accidentes que se producen cada año, especialmente en carreteras convencionales. Los principales factores concurrentes en un accidente de tráfico son el factor humano, el vehículo y la infraestructura. De hecho, la mayor parte de los accidentes se 
producen por la interacción entre el factor infraestructura y el factor humano. Para el análisis de esta interacción el principal parámetro a estudiar es la velocidad de operación. La AASHTO (2011) define la velocidad de operación como aquella velocidad a la que circulan los vehículos ligeros en condiciones de flujo libre. Esta velocidad no es un valor fijo sino que presenta cierta variabilidad, tanto transversal (en una sección de la carretera diferentes conductores desarrollan diferentes velocidades), como longitudinal a lo largo de un recorrido (un mismo conductor desarrolla diferentes velocidades en diferentes puntos de la carretera).

Considerando la dificultad de estudiar toda la distribución de la velocidad en condiciones de flujo libre, suele utilizarse el percentil 85 de la misma en una ubicación concreta o en un elemento de trazado concreto, especialmente para su diseño geométrico. Sin embargo, algunos estudios (Fitzpatrick et al., 2003) han puesto de manifiesto la necesidad en el diseño y la explotación de carreteras de considerar la distribución completa de velocidades en lugar de solamente un percentil. De hecho, Figueroa y Tarko (2005) y Porter et al. (2012) mostraron cómo es posible que una carretera con una velocidad media alta y una baja variabilidad presente el mismo percentil 85 que una carretera con una media mucho más baja pero con una alta variabilidad.

Por otra parte, considerando la ocurrencia de accidentes y la consistencia, Garber y Gabiraju (1989) concluyeron que la tasa de accidentes en una carretera no aumenta necesariamente al aumentar la velocidad media pero sí al aumentar su varianza. Además, Collins et al. (1999) destacaron en su estudio que puntos de la carretera con altos valores de variabilidad de velocidades están generalmente asociados con errores de los conductores y que cambios significativos en los parámetros de la distribución de la velocidad pueden ser un signo de inconsistencias en el diseño.

Todo ello confirma la necesidad de considerar no sólo un percentil, sino la distribución completa de velocidades en flujo libre. Su estudio puede basarse en la calibración de modelos para cada percentil (Schurr et al., 2002; Andueza, 2000), o en la calibración de modelos basados en la velocidad media y en la varianza (Collins et al., 1999; Lindeman y Ranft, 1978; Jacob y Anjaneyulu, 2013).

La principal limitación del primer tipo de modelos es que no pueden predecir otro percentil que no sea aquél para el que han sido calibrados. Por el contrario, los modelos basados en la media y la varianza pueden estimar cualquier percentil, siempre y cuando la distribución de la velocidad sea conocida. De hecho, como se destaca en Taylor et al. (1996) una hipótesis común en la teoría del flujo del tráfico es que las velocidades en flujo libre se distribuyen según una distribución normal, permitiendo así el cálculo de manera sencilla de los percentiles de velocidad de operación.

Los estudios cuyo objetivo es la calibración de modelos para la estimación de diferentes percentiles de la velocidad se centran normalmente en la media, el percentil $85 \mathrm{y}$ el percentil 
95. En ellos, el principal inconveniente son los parámetros usados en los modelos, ya que generalmente las variables independientes son diferentes para cada modelo. Un ejemplo son los modelos desarrollados por Schurr et al. (2002) en los que la velocidad media depende del ángulo de deflexión, la longitud del arco de la curva y la velocidad límite, mientras que el percentil 85 depende del ángulo de deflexión, la longitud del arco de la curva y de la pendiente de entrada.

Por otra parte, los resultados de los estudios basados en la media y la desviación típica de la distribución de velocidades sugieren que la desviación típica de la velocidad aumenta con el radio para curvas de radio pequeño y permanece constante para curvas de radio mayor. Al aumentar la desviación al aumentar el radio, aumenta también al aumentar la velocidad (Collins et al., 1999; Lindeman y Ranft, 1978). Además, Jacob y Anjaneyulu (2013) concluyeron que hay cambios en la distribución de la velocidad de los vehículos entre la recta y el centro de la curva. Había una reducción de velocidad desde la recta hasta el centro de la curva y la variabilidad en el centro de la curva era menor que en la recta. Calibraron modelos que mostraban que tanto la velocidad media como la desviación típica aumentaban con el radio y disminuían conforme la longitud de la curva aumentaba.

Una variación de esta metodología es la calibración de una ecuación para estimar todos los percentiles. Figueroa y Tarko (2005) calibraron un modelo para estimar todos los percentiles de la velocidad. La ecuación que permitía la estimación de la velocidad en curvas incluía cuatro variables diferentes. La primera constante y los cinco siguientes términos se corresponden con la velocidad media mientras que la segunda constante y las dos variables siguientes, cuyos nombres empiezan por Zp se corresponden con la desviación típica.

Lobo et al. (2014) calibró un modelo frontera de velocidad en flujo libre. Este modelo permite la estimación de la velocidad del vehículo que circula más rápido en condiciones de flujo libre en función de las características geométricas del trazado, y, a partir de él, la estimación de todos los percentiles de la velocidad.

Como se ha mencionado anteriormente, considerando los análisis estadísticos en los que se basan estos estudios, tienen que basarse en la hipótesis de que las velocidades se distribuyen según una normal para una localización específica. Sin embargo, los test de normalidad casi nunca están incluidos en los artículos.

Lindeman y Ranft (1978), tras la observación de 10 curvas, dieron valores de asimetría y curtosis de la distribución de velocidades, confirmando que la velocidad no se distribuye según una normal.

Fitzpatrick et al. (2000) también comprobaron si los datos se distribuían según una normal, usando en este caso el test Shapiro-Wilk al considerar que las velocidades siguen una distribución continua, así como también gráficos como stem-and-leaf plot y normal 
probability plot. Los resultados de estos tests indicaron que en la mayoría de las localizaciones las velocidades se distribuían según una normal. En aquellas localizaciones en las que los test de normalidad dieron negativo, se realizó un estudio adicional basado en el normal probability plot que sí indicaron una distribución normal. De esta forma, en la mayoría de los casos se asumió que la velocidad se ajustaba a una distribución normal.

En la misma línea, Fazio et al. (2014) llevó a cabo el test chi-square goodness-of-fit para cada una de las localizaciones, con el fin de determinar si la distribución de las velocidades observadas en ellas seguían una distribución normal o no, concluyendo que solo seis de diez presentaban una distribución normal de velocidades.

A pesar de los estudios mencionados en los párrafos anteriores, la hipótesis de que la velocidad en flujo libre sigue una distribución normal es ampliamente asumida, incluso sin comprobarla (Figueroa y Tarko, 2005; Lobo et al., 2014). De hecho, en muchos casos se asume esta hipótesis para el análisis estadístico sin mencionar cómo se distribuyen los datos y no se han estudiado otras distribuciones que pudieran ajustar mejor el comportamiento de la velocidad en flujo libre.

\section{OBJETIVOS E HIPÓTESIS}

El principal objetivo de esta investigación es analizar la distribución de la velocidad en flujo libre en curvas de carreteras convencionales, con especial énfasis en la media y la desviación típica. Asimismo, se ha analizado si la distribución normal se ajusta adecuadamente a la distribución real de velocidades en una ubicación concreta o no.

La hipótesis en la que se basa este estudio es que tanto la media como la desviación típica está altamente correlacionadas con las características geométricas del diseño de la carretera. Además, aunque tradicionalmente se ha asumido que la velocidad se distribuye según una normal, es posible que otras distribuciones estadísticas reflejen mejor el comportamiento de los conductores.

\section{METODOLOGÍA}

Esta investigación se basa en perfiles continuos de velocidad en flujo libre de más de 16.000 $\mathrm{vh} \cdot \mathrm{km}$ obtenidos a partir de campaña de toma de datos desarrollada en 2008 en Valencia (España). Todas las jornadas de toma de datos se realizaron en días laborables entre las 8:30 y las 14:00 y en condiciones meteorológicas favorables. Para ello, se siguió la metodología de toma de datos desarrollada por Pérez-Zuriaga et al. (2010).

Los tramos de estudio consistieron en 10 tramos de carretera convencional con longitudes variables entre 5 y $20 \mathrm{~km}$, con una Intensidad Media Diaria (IMD) que variaba entre 850 y 7.000 vh/día (Pérez-Zuriaga et al., 2010). 
Para la toma de datos se instalaron dos controles en cada uno de los extremos de los tramos de estudio, de forma que cada vehículo que accedía al tramo era detenido y se le animaba a participar en el estudio. Si el conductor daba su consentimiento, se le hacían una serie de preguntas sociodemográficas y se instalaba un GPS de $1 \mathrm{~Hz}$ en el techo del vehículo. Considerando que la participación en el estudio podía modificar el comportamiento de los conductores, se llevó a cabo una comprobación con el fin de determinar si la observación podía considerarse naturalística o no (Pérez-Zuriaga et al., 2013), concluyendo con la validación de la metodología al no encontrar diferencias significativas entre las velocidades de los vehículos que circulaban por los tramos de estudio días previos a la toma de datos y las velocidades de los vehículos durante la toma de datos.

Además del carácter naturalístico de la toma de datos, fue necesario también comprobar que los vehículos circulaban en condiciones de flujo libre, para en caso de encontrar algún vehículo que en algún tramo circulara condicionado por otros vehículos no considerar esa parte de su perfil en el estudio. La metodología utilizada para la evaluación de las condiciones de circulación en flujo libre, desarrollada por Pérez-Zuriaga et al. (2013), está basada en la hipótesis de que cada conductor se comporta de una forma particular, aproximándose su perfil de velocidad individual al de un percentil. Por tanto, cuando el perfil individual de un conductor se aleja bruscamente del percentil próximo se considera que está condicionado. Tras eliminar los tramos de los perfiles de velocidad individuales en los que la circulación no se producía en condiciones de flujo libre, la muestra de conductores varía entre 53 y 121 conductores, dependiendo del elemento.

La geometría de los tramos de carretera utilizados en el estudio se ha restituido mediante el algoritmo desarrollado por Camacho-Torregrosa et al. (2015) basado en el azimut.

Una vez estudiada la geometría de los tramos de estudio y los perfiles continuos de velocidad desarrollados por los conductores en estos tramos, se seleccionaron 63 curvas aisladas, evitando incluir en el estudio curvas compuestas. De las curvas seleccionadas se tomó la velocidad en el centro de las mismas, tras comprobar que no había diferencias significativas entre la velocidad mínima en las curvas y la velocidad registrada en este punto.

La Tabla 1 resume las principales características de los elementos geométricos considerados en esta investigación.

\begin{tabular}{|l|c|c|c|c|}
\hline & Radio $(\mathrm{m})$ & Longitud $(\mathrm{m})$ & $\begin{array}{c}\text { Velocidad media } \\
(\mathrm{km} / \mathrm{h})\end{array}$ & $\begin{array}{c}\text { Desviación típica de la } \\
\text { velocidad }(\mathrm{km} / \mathrm{h})\end{array}$ \\
\hline mínimo & 52 & 93 & 48 & 6 \\
\hline máximo & 645 & 425 & 96 & 13 \\
\hline
\end{tabular}

Tabla 1 - Resumen de las características de las curvas 


\section{DISTRIBUCIÓN DE VELOCIDAD EN CURVAS}

El tratamiento de los datos de geometría y de velocidad, obtenidos de la toma de datos y de la restitución geométrica, dio como resultado una base de datos en los que para cada curva se dispone de sus características geométricas, la velocidad en flujo libre individual de cada uno de los conductores y la media y la desviación típica de la distribución de velocidad en flujo libre.

\subsection{Media y desviación típica de la distribución de velocidad en curvas.}

La variable geométrica con más influencia en la elección de la velocidad desarrollada por los conductores en curva es el radio de la misma, según todas las investigaciones anteriores. Por ello, es este el parámetro elegido para el estudio de la media y la desviación típica de la distribución de la velocidad en flujo libre en esta investigación.

La Figura 1 muestra cómo la velocidad en flujo libre media varía aumentando conforme aumenta el radio de la curva. Como se esperaba, las curvas con radio más pequeño presentan velocidades más bajas, mientras que curvas con radios mayores presentan mayores velocidades.

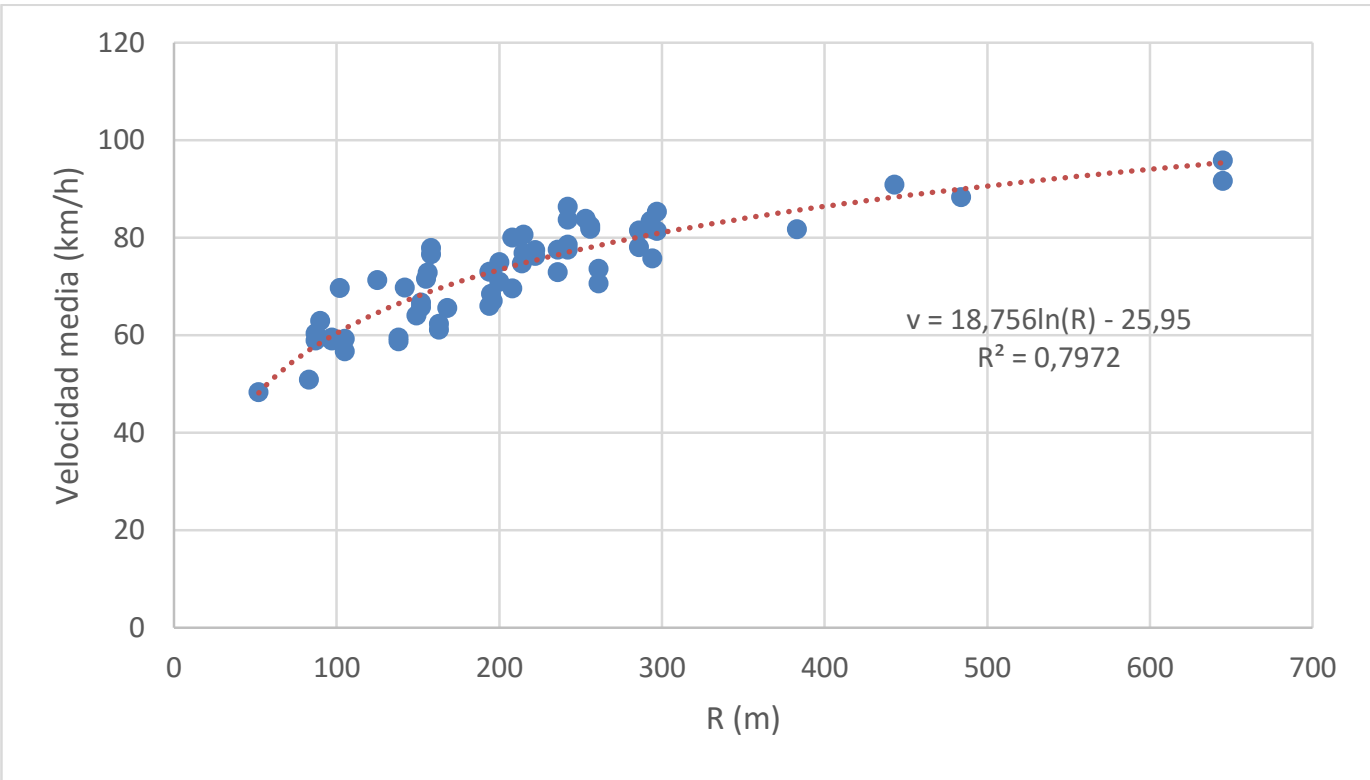

Fig. 1 - Velocidad media vs radio

Como puede observarse en la Figura 1, la relación entre la velocidad media y el radio no es lineal, ya que para radios altos la velocidad se aproxima a una constante. Esta relación se debe a que radios altos suponen un control geométrico menor lo que anima a los conductores a circular a mayor velocidad, como ocurriría en las rectas.

La Figura 2 muestra la relación entre la variabilidad de la velocidad en flujo libre y el radio. Como puede observarse esta variabilidad aumenta con el radio, siguiendo una forma 
funcional hiperbólica. Al igual que se ha observado en el caso de la velocidad media, los valores de la desviación típica se estabilizan para grandes radios, donde el control geométrico es muy débil y los conductores se comportan como lo harían en rectas.

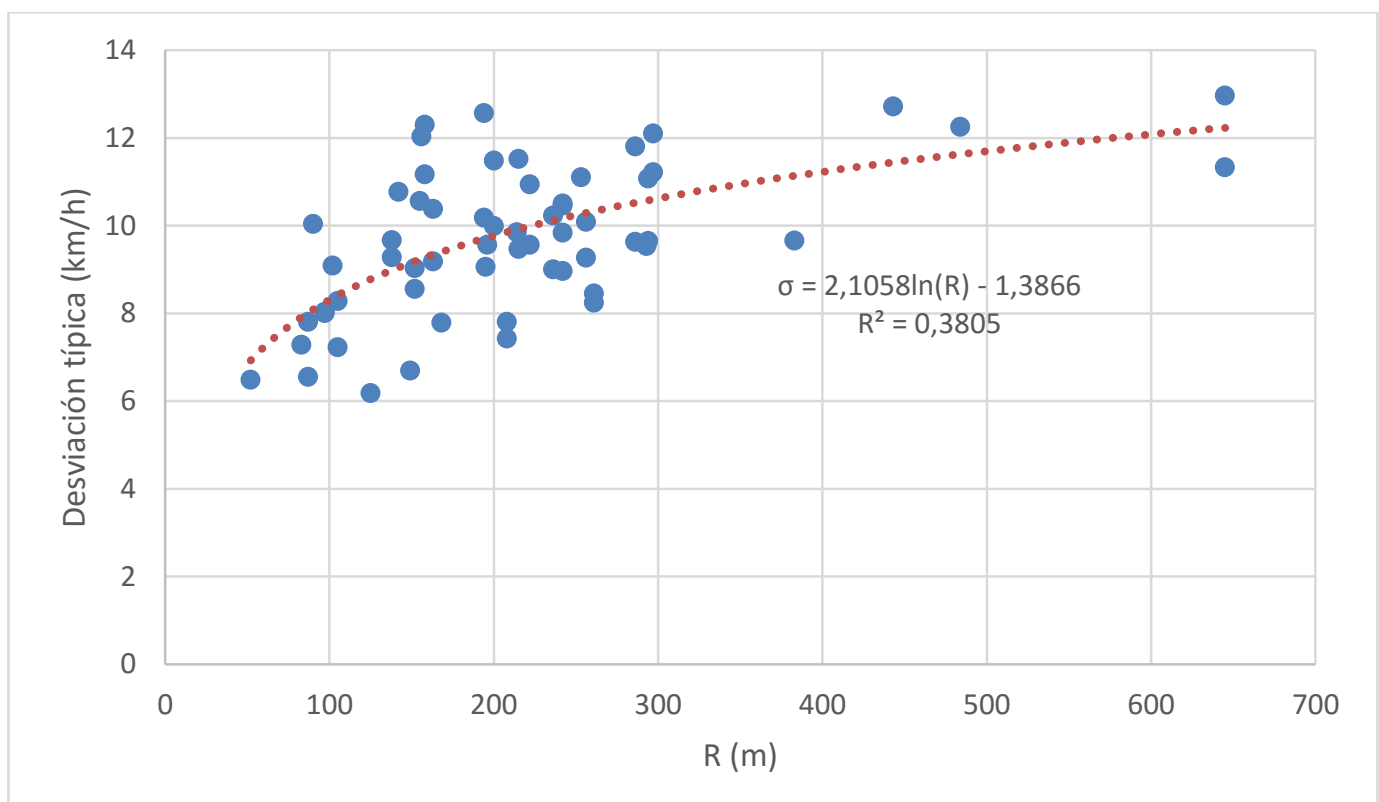

Fig. 2 - Desviación típica vs radio

Por tanto, tanto la media como la desviación típica de la distribución de velocidades en flujo libre están altamente relacionadas con el radio de la curva. Esta conclusión podría indicar que ambos parámetros están también relacionados. La Figura 3 muestra cómo la variabilidad de la velocidad en flujo libre varía con la velocidad media en flujo libre.

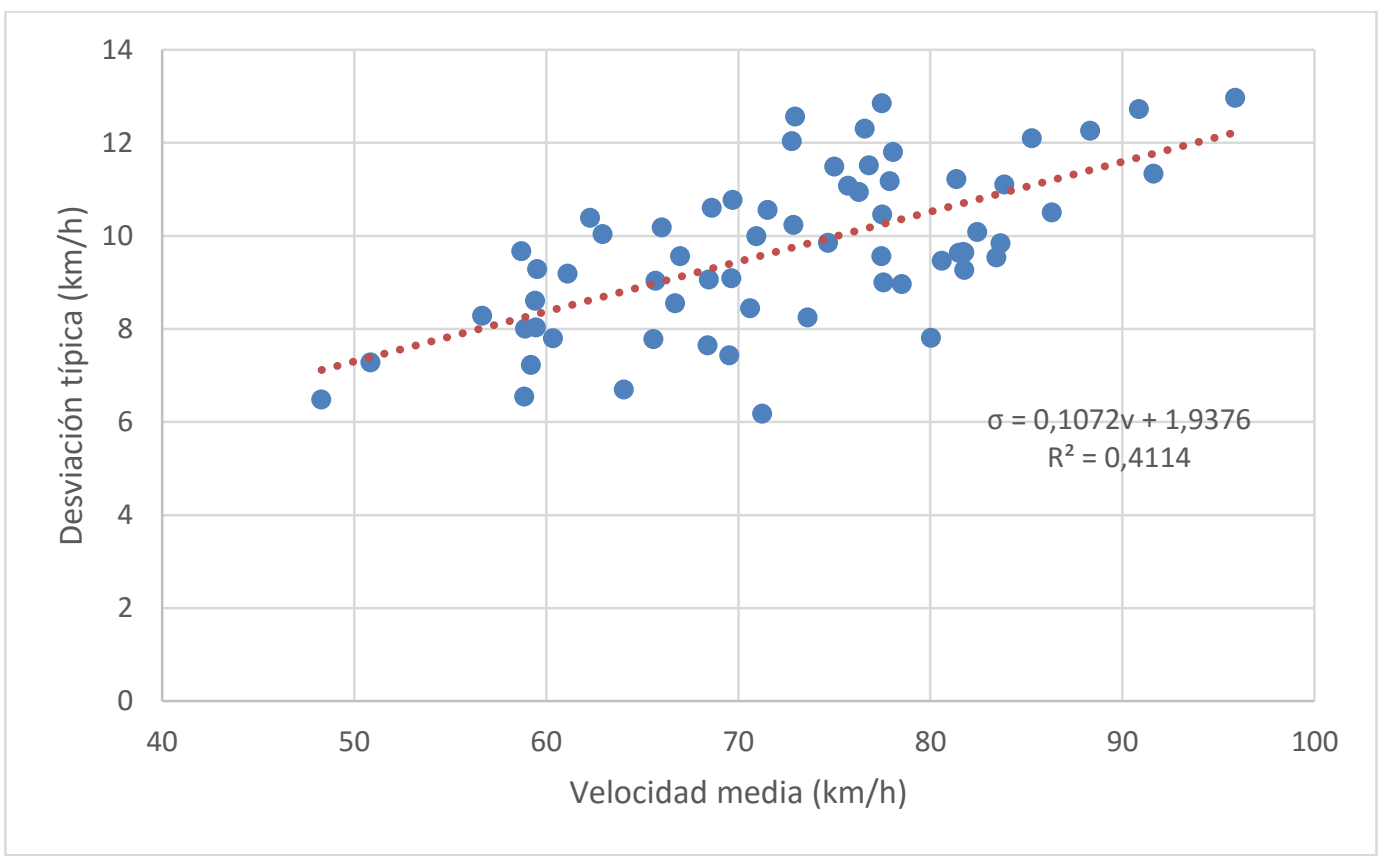

Fig. 3 - Desviación típica vs Velocidad media 
Esta relación parece ser lineal con pendiente positiva. En este caso, no se observa una estabilización para altos valores de la velocidad media.

\subsection{Estudio de la normalidad de los datos.}

Tras el análisis de la media y la variabilidad de la distribución de la velocidad en flujo libre, se han llevado a cabo algunos test de normalidad con el fin de comprobar si se puede asumir que la distribución de velocidad se comporta según una distribución normal.

Para llevar a cabo una prueba de normalidad existen diferentes test que pueden utilizarse. Teniendo en cuenta que ninguno de ellos puede considerarse como absolutamente concluyente, se ha considerado la combinación de cuatro test: prueba del Chi-cuadrado, prueba de Shapiro-Wilk, prueba de sesgo estandarizado y prueba de curtosis estandarizada. Para ellos, se ha considerado un nivel de confianza de $95 \%$.

Los citados test tienen las siguientes características:

- La prueba de Chi-cuadrado comprueba la normalidad de los datos contrastando las frecuencias observadas con las frecuencias esperadas de acuerdo a una distribución normal. Un p-value inferior a 0,05 indica que los datos no se ajustan a una distribución normal.

- La prueba de Shapiro-Wilk asume como hipótesis nula una distribución normal de los datos. Un p-value inferior a 0,05 indica que los datos no se ajustan a una distribución normal.

- La prueba de sesgo estandarizado mide el grado de asimetría de la distribución alrededor de su media. Un sesgo estandarizado dentro del rango $[-2,+2]$ indica una distribución simétrica, que es condición necesaria pero no suficiente para asumir una distribución normal.

- La prueba de curtosis estandarizada mide el grado de concentración de la distribución. Como en la anterior prueba, es necesario un valor dentro del rango $[-2,+2]$ para poder asumir una distribución normal, aunque otras distribuciones también precisan esta condición.

La Tabla 2 muestra los resultados de este análisis. En la mayoría de las curvas (sombreadas) las velocidades no siguen una distribución normal, de acuerdo con las pruebas de normalidad estudiadas. Por tanto, no puede asumirse que la velocidad en flujo libre se comporte según una distribución normal en curvas de carreteras convencionales, aunque en algunos casos puede considerarse una buena aproximación. De hecho, según el análisis realizado, en 46 de 63 curvas las velocidades no siguen una distribución normal: 36 según la prueba de Chicuadrado, 30 según la prueba Shapiro-Wilk, 14 según la prueba de sesgo estandarizado y 17 según la prueba de curtosis estandarizada. 


\begin{tabular}{|c|c|c|c|c|c|c|c|c|}
\hline Curve & $\begin{array}{c}\text { Chi- } \\
\text { cuadrado }\end{array}$ & P-Value & Shapiro-Wilk & P-Value & Sesgo est. & P-Value & Curtosis est. & P-Value \\
\hline C01I01 & 46.14930 & 0.0002 & 0.97072 & 0.2846 & 0.70402 & 0.48142 & -0.85670 & 0.39161 \\
\hline C01IO2 & 11.63640 & 0.6354 & 0.95255 & 1056 & 1.40538 & 0.15991 & 1.28926 & 0.19731 \\
\hline C01IO3 & 15.43280 & 0.6320 & .96234 & 1076 & 1.79787 & 0.07220 & 3.09282 & 0.00198 \\
\hline C01IO4 & 20.42860 & 0.1561 & 0.98784 & 0.9523 & 0.23647 & 0.81307 & -0.37737 & 0.70590 \\
\hline C01I05 & 21.07460 & 0.2756 & 0.96666 & 0.1813 & 0.90849 & 0.36362 & -0.30375 & .76131 \\
\hline C01IO6 & 13.55220 & 0.7577 & 0.97558 & 0.4557 & 0.90499 & 0.36547 & -0.14474 & .88491 \\
\hline C01V01 & 27.29410 & 0.1271 & 0.96069 & 0.044 & 0.18410 & 0.85393 & -1.14748 & 0.25118 \\
\hline C01V02 & 44.87500 & 0.0000 & 0.88203 & 0.0019 & 0.10574 & 0.91579 & 1.02108 & 0.30722 \\
\hline C03I01 & 63.90530 & 0.0000 & 0.93722 & 0.0002 & 2.94361 & 0.00324 & 3.55024 & 0.00038 \\
\hline $\mathrm{CO3I02}$ & 51.27370 & 0.0002 & & 0.0000 & & 0.00273 & & 00193 \\
\hline C03V01 & 28.00 & 0.1094 & 0.922 & & & 0.00133 & & 00016 \\
\hline C03V02 & 57.2 & 0.0000 & 0.92944 & 0.0001 & 3047 & 0.00632 & 26 & 0.00229 \\
\hline C04IO1 & 27.49060 & 0.1931 & .96363 & 0.0374 & 1.70871 & 0.08750 & 85 & 0.52879 \\
\hline C04IO2 & 43.18180 & 0.0044 & 0.95433 & 0.0039 & 1.89397 & 0.05823 & 0.96219 & 33595 \\
\hline C04IO3 & 28.11110 & 0.1720 & 0.95380 & 0.0038 & 2.06317 & 0.03910 & 1.67478 & 0.09398 \\
\hline C04IO4 & 51.88240 & 0.0002 & 0.95353 & 0.0051 & 1.70047 & 0.08904 & 1.38464 & 16616 \\
\hline C04V01 & 24.10260 & 0.1922 & 0.98430 & 0.7957 & 0.38866 & 0.69752 & 0.37088 & 0.71072 \\
\hline C04V02 & 32.30120 & 0.0289 & 0.97281 & 0.2755 & 0.01210 & 0.99034 & -1.12822 & 0.25923 \\
\hline C04V03 & 59.76470 & 0.0000 & & 0.0418 & & 0.08190 & & 0.08935 \\
\hline C05IO & 53.70270 & 0.0000 & 4 & & & 0.22731 & & 20620 \\
\hline C05I02 & 72.43240 & 0.0000 & 28 & 0.7194 & & 0.46441 & & 56075 \\
\hline C05I03 & 16.84510 & 0.5337 & 0.96407 & 0.1179 & 1.38454 & 0.16619 & & 27121 \\
\hline C05I04 & 31.13040 & 0.0278 & 0.92215 & 0.0002 & 2.55730 & 0.01055 & 2.61874 & 0.00883 \\
\hline C05I05 & 27.73530 & 0.0662 & 0.94876 & 0.0160 & 2.11249 & 0.03464 & 2.14919 & .03162 \\
\hline C05V01 & 45.42720 & 0.0015 & 0.95313 & 0.0044 & 2.68189 & 0.00732 & 3.11997 & 0.00181 \\
\hline C05V02 & 52.25000 & 0.0002 & 0.96808 & 0.0922 & 1.62467 & 0.10423 & 0.69762 & 0.48541 \\
\hline C05V04 & 44.55770 & 0.0030 & .93335 & 0000 & 3.14839 & 0.00164 & 3.76226 & .00017 \\
\hline C05V05 & 40.38 & 0.0066 & & & & 0.003 & & .00165 \\
\hline C06V01 & 47.4 & & & 56 & & 0.14821 & & 08093 \\
\hline C06V02 & 54.1 & & & 0.0010 & 07 & 0.01303 & & 0.00239 \\
\hline C06I01 & 33.50000 & 0.0297 & & 0.4693 & 21879 & 0.22292 & & 0.24089 \\
\hline C07I02 & 14.43640 & 0.5662 & 0.94930 & 0.0393 & 1.70952 & 0.08735 & 1.25476 & 0.20957 \\
\hline C07I03 & 13.03850 & 0.6699 & 0.96878 & 0.3254 & 0.83540 & 0.40349 & -0.45325 & 0.65037 \\
\hline C07IO4 & 26.19230 & 0.0513 & 0.96230 & 0.1805 & 1.08431 & 0.27823 & 0.55368 & 0.57980 \\
\hline C07I05 & 26.92310 & 0.0423 & 0.94395 & .0265 & 1.86198 & 0.06261 & 1.85922 & .06300 \\
\hline C07IO6 & 12.64000 & 0.6300 & 94011 & 0205 & 1.78552 & 0.07418 & 1.52259 & 12786 \\
\hline C07V01 & 52.13700 & 0.0000 & 95728 & 0428 & 1.51849 & 0.12889 & 0.71967 & 0.47172 \\
\hline C07V02 & 113.26800 & 0.0000 & & 0101 & 73162 & 0.08334 & & 0.04101 \\
\hline C07V04 & 31.42470 & 0.0 & & & & 0.0 & & 0.39116 \\
\hline C07V05 & & & & & & & & 426 \\
\hline C07V06 & 24.02940 & & & 1067 & 097 & 807 & & 0.87845 \\
\hline C07V07 & 25.32430 & 0.1162 & 0.97344 & 3376 & 1.03606 & 0.30017 & 0.16115 & 0.87197 \\
\hline C07V08 & 39.21050 & 0.0041 & 0.98034 & 0.6232 & 0.72633 & 0.46764 & 1.55955 & 11887 \\
\hline C08I01 & 59.71430 & 0.0000 & 0.97424 & 0.2703 & 0.93549 & 0.34954 & -0.40957 & 0.68212 \\
\hline C08102 & 48.44900 & 0.0006 & 0.98525 & 0.7997 & 0.45354 & 0.65016 & 0.76237 & 0.44584 \\
\hline C08103 & 31.56840 & 0.0647 & 0.98725 & 0.8830 & 1.06197 & 0.28825 & 1.25284 & 0.21026 \\
\hline C08104 & 58.00000 & 0.0000 & 6923 & 1329 & 1.67031 & 0.09486 & 1.971 & 0.04867 \\
\hline C08V01 & 46.50570 & 0.0006 & 0.97255 & 0.2512 & 1.36988 & 0.17073 & 0.88971 & 0.37362 \\
\hline C08V02 & 53.90800 & 0.0000 & & 0.1628 & 1.34912 & 0.17730 & & 0.60836 \\
\hline C08V03 & 27.47130 & 0.1225 & 0.97909 & 0.5227 & 0.18330 & 0.85456 & & 0.65416 \\
\hline C08V05 & 15.31030 & & 0.97149 & 0.2186 & 0.05398 & 0.95695 & -0.81667 & 0.41412 \\
\hline C08V06 & 14.30590 & 0.8146 & 0.97604 & 0.3902 & 0.36238 & 0.71706 & 1.82303 & 0.06830 \\
\hline C09I01 & 96.59260 & 0.0000 & 0.96342 & 0.1878 & 0.05098 & 0.95933 & -0.73310 & 0.46349 \\
\hline C09IO2 & 13.74550 & 0.6176 & 0.97596 & 0.5434 & 1.14578 & 0.25189 & 2.38569 & 0.01705 \\
\hline & 14.65960 & 0.4762 & 0.97648 & 0.6190 & 0.70177 & 0.48282 & -0.49238 & \\
\hline
\end{tabular}




\begin{tabular}{|c|cc|cc|cc|cc|}
\hline Curve & $\begin{array}{c}\text { Chi- } \\
\text { cuadrado }\end{array}$ & P-Value & Shapiro-Wilk & P-Value & Sesgo est. & P-Value & Curtosis est. & P-Value \\
\hline C09V02 & 27.04000 & 0.0284 & 0.98158 & 0.7842 & 0.17906 & 0.85789 & 1.41388 & 0.15740 \\
C10I01 & 12.29410 & 0.8316 & 0.95396 & 0.0338 & 1.96565 & 0.04934 & 2.13126 & 0.03307 \\
C10I02 & 87.83330 & 0.0000 & 0.90207 & 0.0000 & 2.44000 & 0.01469 & 3.57206 & 0.00035 \\
C10I03 & 57.95240 & 0.0000 & 0.95000 & 0.0261 & 0.68986 & 0.49028 & 1.56166 & 0.11837 \\
C10I04 & 25.76920 & 0.0787 & 0.95981 & 0.0842 & 1.68534 & 0.09192 & 1.74485 & 0.08101 \\
C10V01 & 35.05880 & 0.0039 & 0.93874 & 0.0162 & 1.95010 & 0.05116 & 3.27320 & 0.00106 \\
C10V02 & 27.65380 & 0.0347 & 0.98638 & 0.9188 & 0.09018 & 0.92814 & 1.04645 & 0.29535 \\
C10V03 & 16.24000 & 0.3662 & 0.97471 & 0.5324 & 0.74794 & 0.45449 & -0.27550 & 0.78293 \\
\hline
\end{tabular}

Para completar el análisis se desarrolló para cada curva su correspondiente gráfico de probabilidad normal, mostrando la mayor parte de las curvas asimetría positiva en su distribución de velocidades.

\section{DISCUSIÓN}

El análisis de los datos de velocidad tomados en los tramos de carretera en estudio ha mostrado cómo la media de la distribución de velocidad de vehículos ligeros circulando en condiciones de flujo libre en curvas de carreteras convencionales y su desviación típica están relacionadas y dependen a su vez de las características geométricas de las curvas.

La relación entre la media y el radio de la curva y la desviación típica y este parámetro es similar. En curvas con radio reducido tanto la media como la desviación son bajas, debido probablemente al control geométrico que impone este elemento del diseño que hace que la elección de la velocidad por parte del conductor esté limitada. Tanto la velocidad media como la desviación típica aumentan con el radio, hasta llegar a un punto en que permanecen prácticamente constante. Esta situación ocurre en curvas con radio amplio donde el comportamiento es similar al que se produce en rectas. En ellas el control geométrico es mínimo y la elección de la velocidad por parte del conductor depende en mayor medida de las características de este.

Por otra parte, se ha comprobado la normalidad de los datos, concluyendo que en un $73 \%$ de las curvas no se puede asumir que los valores de la velocidad registrados se distribuyan según una distribución normal.

A la vista de los resultados se planteó la cuestión de si esta falta de normalidad podía ser debida a las características de los conductores, considerando que la muestra pudiera estar de alguna forma sesgada, o si podía tener relación con la geometría de las curvas.

Por ello, se hizo un análisis en profundidad de los factores sociales de los conductores, de los que se tenían datos gracias a las encuestas que se realizaron al colocar los dispositivos GPS en los vehículos. Estos factores incluían datos sociales, experiencia en la conducción, tipo de vehículo, motivo del viaje y características del entorno y de la infraestructura. De 
este estudio se concluyó que no hay diferencias significativas entre los conductores que circulan por los elementos geométricos en los que la velocidad se distribuye según una normal y los que circulan por curvas en los que la velocidad no sigue esta distribución. Es más, incluso curvas con características geométricas similares en un mismo tramo con los mismos conductores se comportaban respecto a la normalidad de forma diferente.

En cuanto a la posibilidad de que este comportamiento diferente frente a la normalidad estuviera condicionado por las características geométricas de las curvas, también se descartó, concluyendo que la falta de normalidad no está asociada específicamente a curvas con radio amplio o reducido (Figura 4).

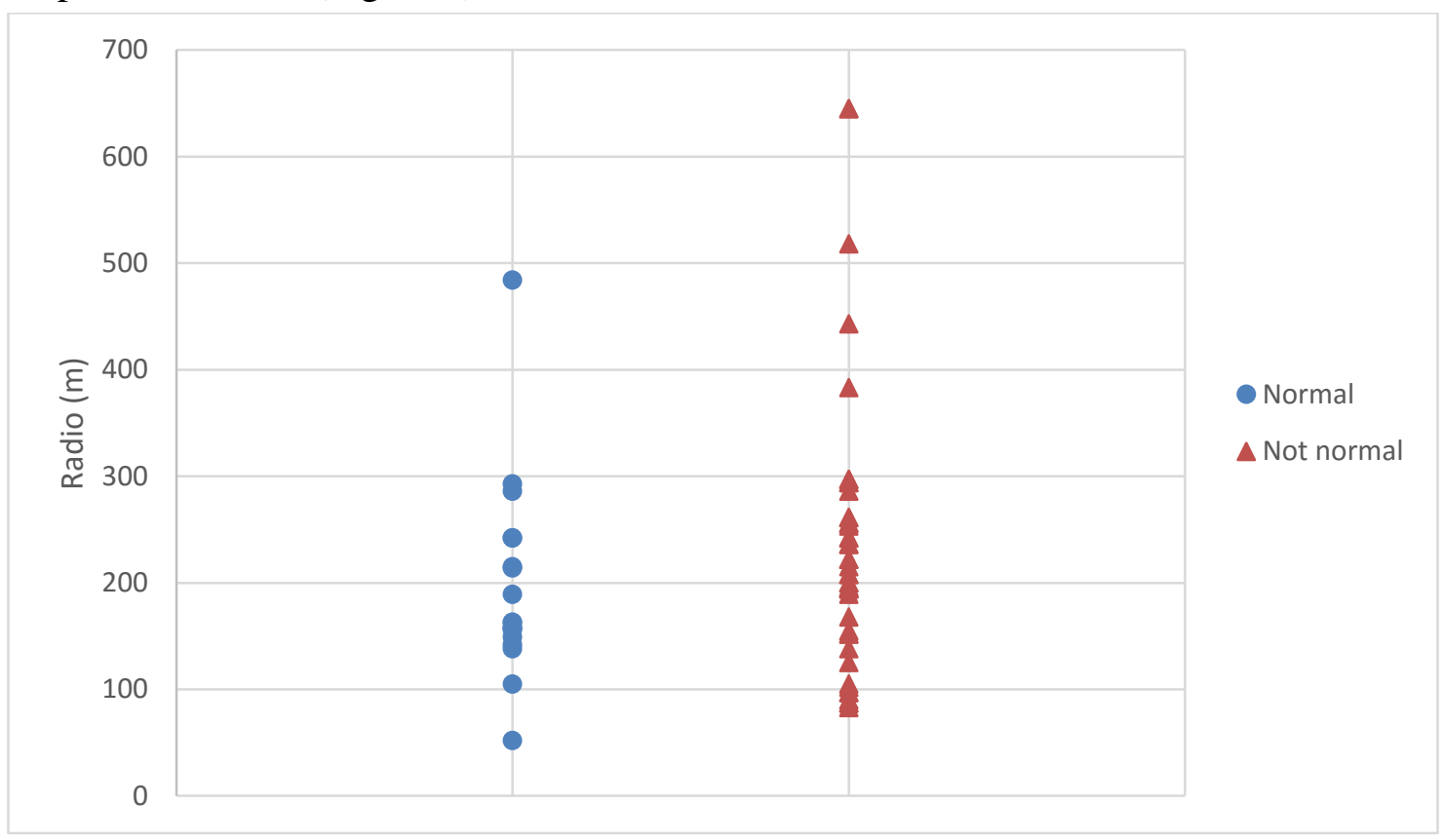

Fig. 4 - Distribución por radios

Teniendo en cuenta estos resultados, se recomienda llevar a cabo un mayor análisis en profundidad con el fin de determinar que distribución estadística se ajusta mejor a la distribución de velocidades en flujo libre.

\section{CONCLUSIONES}

En este artículo se ha presentado un análisis en profundidad de la distribución de la velocidad desarrollada por vehículos ligeros en flujo libre en 63 curvas de carreteras convencionales. Para ello, se ha estudiado tanto el comportamiento de su velocidad media como de su desviación típica. Asimismo, se ha comprobado la hipótesis de normalidad de los datos, ampliamente utilizada en los estudios relacionados.

La toma de datos en la que se ha basado esta investigación se ha desarrollado según la metodología propuesta por Pérez-Zuriaga et al. (2010), mediante la que es posible obtener el perfil continuo de velocidad de cada uno de los conductores que circulan por un tramo de 
carretera.

El análisis de la distribución de velocidades de vehículos ligeros en condiciones de flujo libre se ha basado en el estudio del comportamiento de la velocidad media y de la desviación típica en función del radio. Como se esperaba, la velocidad media en curvas está altamente relacionada con el radio, al igual que la variabilidad. Esta relación no es lineal, sino que se aproxima a una velocidad constante para radios amplios, comportándose estas curvas como rectas.

Adicionalmente, se ha comprobado la hipótesis de normalidad de velocidad en flujo libre para cada una de las curvas estudiadas. Las pruebas estadísticas realizadas (prueba de Chicuadrado, prueba de Shapiro-Wilk, prueba de sesgo estandarizado, prueba de curtosis estandarizada) han dado como resultado que en la mayoría de las curvas las velocidades no siguen una distribución normal. Esta falta de normalidad no parece estar relacionada ni con la geometría de las curvas ni con las características de los conductores.

Estos resultados pueden sentar las bases para futuras investigaciones en las que se estudie qué distribución estadística puede ajustarse mejor al comportamiento de los conductores y con ella calibrar modelos que permitan estimar no sólo un percentil de la distribución de velocidades de vehículos ligeros circulando en condiciones de flujo libre, sino la distribución completa.

\section{AGRADECIMIENTOS}

El estudio que se ha presentado es parte del proyecto de investigación "CASEFU - Estudio experimental de la funcionalidad y seguridad de las carreteras convencionales" (TRA201342578-P), subvencionado por el Ministerio de Economía y Competitividad y el Fondo Social Europeo. Asimismo, los autores quieren agradecer al Centro de Estudios y Experimentación de Obras Públicas (CEDEX) del Ministerio de Fomento por subvencionar la investigación en la que se llevó a cabo la toma de datos, a la Dirección General de Obras Públicas, Transporte y Movilidad de la Conselleria de Vivienda, Obras Públicas y Vertebración del Territorio, a la Diputación de Valencia, y al Ministerio del Interior, especialmente a la Dirección General de Tráfico, por su colaboración en la toma de datos.

\section{REFERENCIAS}

AASHTO (2011). A Policy on Geometric Design of Highways and Streets. American Association of State Highway and Transportation, EEUU.

ANDUEZA, P. J. (2000). Mathematical models of vehicular speed on mountain roads. Transportation Research Record: Journal of the Transportation Research Board, vol. 2, No. 1701, pp. $104-110$.

CAMACHO-TORREGROSA, F. J., PÉREZ-ZURIAGA, A. M., CAMPOY-UNGRÍA, J. M., GARCÍA, A. y TARKO, A. P. (2015). Use of Heading Direction for Recreating the 
Horizontal Alignment of an Existing Road. Computer-Aided Civil and Infrastructure Engineering, Vol. 30(4), pp. 282-299.

COLLINS, J., FITZPATRICK, K., BAUER, K. M. y HARWOOD, D. W. (1999). Speed Variability on Rural Two-Lane Highways. Transportation Research Record: Journal of the Transportation Research Board, vol. 1658, pp. 60-69.

FAZIO, J., WIESNER, B. N. y DEARDOFF, M. D. (2014). Estimation of free-flow speed. Journal of Civil Engineering, vol. 18, pp. 646-650.

FIGUEROA, A. M. y TARKO, A. P. (2005). Speed factors on two-lane rural highways in free-flow conditions. Transportation Research Record: Journal of the Transportation Research Board, vol. 1912, pp 39-46.

FITZPATRICK, K., CARLSON, P., BREWER, M. y WOOLDRIDGE, M. D. (2003). Design speed, operating speed and posted speed relationships. Transportation Research Board, NCHRP Report 504.

FITZPATRICK, K. y COLLINS, J. M. (2000). Speed-Profile Model for Two-Lane Rural Highways. Transportation Research Record: Journal of the Transportation Research Board, vol. 1737, pp. 42-49.

GARBER, N. J. y GADIRAJU, R. (1989). Factors affecting speed variance and its influence on accidents. Transportation Research Record: Journal of the Transportation Research Board, vol. 1213, pp. 64-71.

JACOB, A. y ANJANEYULU M. V. L. R. (2013). Operating Speed of Different Classes of Vehicles at Horizontal Curves on Two-Lane Rural Highways. Journal of Transportation Engineering, Vol. 139, pp. 287-294.

LINDEMAN, H. y RANFT, B. (1978). Speed on curves. ETHZ Institute for Traffic Planning and Transport Engineering. EDI Research Contract 3/77, Zurich.

LOBO, A., RODRIGUES, C. y COUTO, A. (2014). Estimating percentile speeds from a maximum operating speed frontier. Transportation Research Record: Journal of the Transportation Research Board, vol. 2404, pp. 1-17.

PÉREZ-ZURIAGA, A.M., GARCÍA, A., CAMACHO-TORREGROSA, F.J. y D'ATTOMA, P. (2010). Modeling Operating Speed and Deceleration on Two-Lane Rural Roads with Global Positioning System Data. Transportation Research Record: Journal of the Transportation Research Board, vol. 2171, pp. 11-20.

PÉREZ-ZURIAGA, A. M., CAMACHO-TORREGROSA, F. J., GARCÍA, A. y CAMPOYUNGRÍA, J. M. (2013). Application of global positioning system and questionnaires data for the study of driver behaviour on two-lane rural roads. IET Intelligent Transport Systems, vol. 7 (2), pp. 182-189.

PORTER, R., DONNELL, E. y MASON, J. (2012). Geometric Design, Speed, and Safety. Transportation Research Record: Journal of the Transportation Research Board, vol. 2309, pp. 39-47. 
SCHURR, K. S., MCCOY, P. T., PESTI, G. y HUFF, R. (2002). Relationship of design, operating, and posted speeds on horizontal curves of rural two-lane highways in Nebraska. Transportation Research Record: Journal of the Transportation Research Board, vol. 1796, pp. $60-71$.

TAYLOR, M., YOUNG, W. y BONSALL, P. W. (1996). Understanding traffic systems: data, analysis and presentation. Routledge. 